\title{
Potencial neuroprotetor, antioxidante e anti-inflamatório do Canabidiol: relevância e perspectivas para o tratamento de doenças neurodegenerativa
}

\author{
Potential neuroprotective, antioxidant and anti-inflammatory of Cannabidiol: relevance \\ and prospects for the treatment of neurodegenerative diseases.
}

Leandro Ebling Flores ${ }^{1}$, Lauren Lúcia Zamin²*

${ }^{1}$ Acadêmico de Ciências Biológicas-licenciatura, Universidade Federal da Fronteira Sul - UFFS / RS; ${ }^{2}$ Professora

Adjunta da UFFS, Campus Cerro Largo.

\begin{abstract}
Resumo
Introdução: a incidência de doenças neurodegenerativas tem aumentado nos últimos anos, passando a constituir um problema de saúde pública. Frente a isso se faz necessário o desenvolvimento de novas modalidades terapêuticas visando evitar, recuperar ou impedir as alterações celulares que possam levar à morte neuronal. Objetivo: realizar uma revisão bibliográfica acerca do potencial neuroprotetor, antioxidante e anti-inflamatório do Canabidiol (CBD) investigando sua ação sobre processos neurodegenerativos associados à doença de Alzheimer, Parkinson e Huntington. Metodologia: foi realizado um estudo descritivo e qualitativo desenvolvido na forma de revisão bibliográfica onde foram utilizados artigos disponíveis nos bancos de dados PubMed, Scielo e Scopus com o período de referência compreendido entre os anos de 1998 e 2016. Resultados: foram selecionados 10 artigos que tratavam do tema e todos eles mostraram que o CBD induziu neuroproteção e possuiu propriedades antioxidantes e anti-inflamatórios em diferentes modelos de citotoxicidade, estresse oxidativo e neurodegeneração. Conclusão: o CBD pode ser um importante agente terapêutico no tratamento, na prevenção e na reversão de lesões oriundas de doenças neurodegenerativas. Entretanto, há necessidade de reforçar estudos e ensaios em modelos animais antes de transpor o uso do CBD para humanos.
\end{abstract}

Palavras-chave: Neuroproteção. Revisão. Canabinóide.

\begin{abstract}
:
Introduction: the incidence of neurodegenerative diseases has increased in recent years, becoming a public health problem. In view of this, it is necessary to develop new therapeutic modalities in order to avoid, recover or prevent cell changes that may lead to neuronal death. Objective: to perform a literature review on the neuroprotective, antioxidant and anti-inflammatory potential effects of Canabidiol (CBD), investigating its action on neurodegenerative processes associated with Alzheimer's disease, Parkinson's and Huntington's disease. Methodology: a descriptive and qualitative study was developed in the form of a bibliographic review where articles available on PubMed, Scielo e Scopus database with the reference period from 1998 to 2016 were used. Results: ten articles were selected that addressed the theme and they all showed that CBD induced neuroprotection and possessed antioxidant and anti-inflammatory properties in different models of cytotoxicity, oxidative stress and neurodegeneration. Conclusion:CBD may be an important therapeutic agent in the treatment, prevention and reversal of lesions from neurodegenerative diseases. However, there is a need to reinforce studies and trials in animal models prior to transposing CBD to humans

Keywords: Neuroprotection. Review. Cannabinoid.
\end{abstract}

\section{INTRODUÇÃO}

A incidência de casos diagnosticados de doenças neurodegenerativas tem aumentado com o passar do tempo, devido à elevação da expectativa média de vida da população, e também devido à evolução da medicina que, através do desenvolvimento e aplicação de novas tecnologias na área terapêutica, aumenta as chances de que as pessoas atinjam a velhice, fazendo com que cresçam os índices de problemas de saúde relacionados à idade (MAGALHÃES; SANDBERG, 2005).

Correspondente/Corresponding: *Lauren Lúcia Zamin - End: Rua Jacob Reinaldo Haupenthal, 1580, CEP 97900-000, Cerro Largo, RS - Tel: (55) 3359-4224 - E-mail: lauren.zamin@uffs.edu.br
Essas patologias progridem à medida que ocorre a degradação celular de certos grupos de neurônios, o que configura um quadro progressivo de declínio da função cognitiva, afetando a memória, o comportamento, a linguagem e o pensamento dos pacientes. As doenças neurodegenerativas mais decorrentes e comuns são a doença de Alzheimer (DA), a doença de Parkinson (DP) e a doença de Huntington (DH) (NUSSBAUM; MCINNES; WILLARD, 2008).

Estima-se que em 2015 cerca de 46,8 milhões de pessoas apresentavam manifestação clínica de pelo menos alguma dessas patologias. Além disso, se essas doenças continuarem a se expressar na mesma frequência atual, é esperado que a cada 20 anos o número 
de pessoas afetadas dobre, chegando a 131,5 milhões de pessoas em 2050 (THE GLOBAL..., 2015).

Tais doenças têm grande impacto social e, com o passar do tempo, têm se configurado como um problema de saúde pública (JOSEPH et al., 2009), primeiro devido ao fato de que são a quarta principal causa de morte no mundo, ficando atrás apenas dos índices de morte por doenças cardíacas, câncer e acidente vascular cerebral (AVC), e também por fatores relacionados ao contingenciamento econômico pois, somente em 2015, foram gastos 118 bilhões de dólares no custeio financeiro para tratamento dessas doenças em todo o mundo (THE GLOBAL..., 2015).

Dessa forma, nos últimos tempos, uma gama de estudos experimentais e clínicos tem proposto diversas modalidades terapêuticas, visando evitar, recuperar ou impedir as alterações celulares que possam levar a morte neuronal. Tais estudos vêm contribuindo para a elucidação de um conhecimento mais esclarecido dessas patologias, e, além disso, buscam alternativas viáveis e seguras para desenvolver um tratamento focado em retardar ou minimizar a progressão dessas doenças.

Muitas substâncias com propriedades neuroprotetoras, antioxidantes e anti-inflamatórias têm sido descritas e utilizadas em estudos relacionados ao processo de regeneração e plasticidade sináptica, no intuito de evitar a morte neuronal após lesões (PEREZ, 2013). Nesse cenário, o Canabidiol (CBD) vem ganhando destaque, pois um grande número de efeitos farmacológicos tem sido atribuído à molécula (SCUDERI et al., 2009), incluindo efeitos anticonvulsivantes, sedativos, ansiolíticos, antipsicóticos, anti-inflamatórias, antioxidantes e neuroprotetores, assim como efeitos sobre o sistema imunológico e circulatório (ZUARDI, 2008).

O CBD é o principal componente não psicotrópico da Cannabis sativa. Foi isolado na década de 1940 e teve sua estrutura química exata elucidada em 1964 (MECHOULAM et al. 1970). Pelo fato de não apresentar potencial psicotrópico característico dos derivados da maconha, é um dos constituintes bioativos com o maior potencial de uso clínico (CRIPPA et al., 2009).
Nesse sentido, o objetivo deste trabalho foi realizar uma revisão bibliográfica acerca do potencial neuroprotetor, anti-inflamatório e antioxidante do CBD, investigando sua ação sobre doenças neurodegenerativas, no intuito de discutir seu uso e sua relevância clínica em patologias como a DA, a DP e a DH, a fim de descrever padrões atuais de resultados obtidos com o uso de CBD para prevenir ou retardar neurodegeneração.

\section{METODOLOGIA}

Foi realizado um estudo descritivo, qualitativo e desenvolvido na forma de revisão bibliográfica relacionada à investigação da ação neuroprotetora, antioxidante e anti-inflamatória do CBD quando utilizado em modelos biológicos de doenças neurodegenerativas. Foram usados no processo de pesquisa artigos disponíveis nos bancos de dados PubMed, Scielo e Scopus no período compreendido entre os anos de 1998, quando foi publicado o primeiro estudo relatando as propriedades neuroprotetoras do CBD, e 2016. As principais palavras-chave utilizadas na busca de referências foram: "Neurodegenerative diseases and cannabidiol"; "Cannabidiol and neuroprotection"; "Cannabidiol and anti-oxidation". Foi realizada a leitura do título e do resumo dos artigos encontrados, como pré-seleção, para avaliar se a temática abordada no trabalho coincidia com o objetivo desta revisão, e aqueles que não se enquadravam foram descartados. Os critérios de exclusão dos artigos encontrados foram (1) artigos que evidenciassem ações neuroprotetoras, antioxidantes e anti-inflamatórias do CBD, mas não fossem investigados em modelos de doenças neurodegenerativas, (2) artigos de revisão, (3) ano de publicação anterior a 1998 e (4) estudos não disponíveis nos bancos de dados consultados.

\section{RESULTADOS E DISCUSSÕES}

Com base nos critérios iniciais e também nos fatores considerados para exclusão, foram encontrados ao final do processo de busca 10 artigos na literatura que atendiam aos objetivos dessa revisão, dos quais os resultados estão descritos resumidamente no quadro 1.

Quadro 1 - Artigos incluídos nesta revisão.

\begin{tabular}{|c|c|c|c|c|c|}
\hline Estudo & Modelo & Tratamento & Variáveis analisadas & Método utilizado & Resultado \\
\hline $\begin{array}{l}\text { Hampson et } \\
\text { al. (1998) }\end{array}$ & $\begin{array}{l}\text { Modelo de doença de Parkin- } \\
\text { son: Neurônios expostos a } \\
\text { níveis tóxicos de glutamato }\end{array}$ & $\begin{array}{l}\text { Administração de CBD en- } \\
\text { tre } 18 \text { e } 20 \text { h após a expo- } \\
\text { sição tóxica ao glutamato. } \\
(2-4 \mu \mathrm{M})\end{array}$ & $\begin{array}{l}\text { Estimulação de receptores } \\
\text { NMDAR, AMPA, e presença } \\
\text { de ROS. }\end{array}$ & Espectrofluorimetria & $\begin{array}{l}\text { CBD evitou a morte celular, } \\
\text { mediando à expressão de } \\
\text { receptores como NMDAR, } \\
\text { AMPA e ROS. }\end{array}$ \\
\hline $\begin{array}{l}\text { luvone et al. } \\
(2004)\end{array}$ & $\begin{array}{l}\text { Modelo de doença de Alzhei- } \\
\text { mer: toxicidade induzida pelo } \\
\text { peptídeo } \beta \text {-amilóide em células } \\
\text { PC12. }\end{array}$ & $\begin{array}{l}\text { Tratamento com CBD } \\
\text { antes da exposição ao } \\
\beta \text {-peptídeo }\end{array}$ & $\begin{array}{l}\text { Presença de ROS, peroxida- } \\
\text { ção lipídica, expressão de } \\
\text { Caspase } 3 \text { e fragmentação } \\
\text { do DNA. }\end{array}$ & $\begin{array}{l}\text { Técnica de Western } \\
\text { Blotting }\end{array}$ & $\begin{array}{l}\text { CBD diminuiu a produção de } \\
\text { ROS, a peroxidação lipídica e } \\
\text { os níveis de Caspase } 3 \text {. }\end{array}$ \\
\hline $\begin{array}{l}\text { Esposito et al. } \\
\text { (2006) }\end{array}$ & $\begin{array}{l}\text { Modelo de Doença de Alzhei- } \\
\text { mer (in vivo): inoculação de } \\
\text { peptídeos } \beta \text {-amilóides humanos } \\
\text { no hipocampo dorsal dos ratos }\end{array}$ & $\begin{array}{l}\text { Administração de CBD } \\
\text { - doses de } 2,5 \text { e } 10 \mathrm{mg} / \\
\mathrm{kg} \text { - durante } 7 \text { dias. }\end{array}$ & $\begin{array}{l}\text { A presença de mRNA para a } \\
\text { GFAP, a expressão de GFAP, } \\
\text { iNOS e IL-1 } \beta \text {. }\end{array}$ & $\begin{array}{l}\text { Análise de imunofluo- } \\
\text { rescência e hibridização } \\
\text { in situ }\end{array}$ & $\begin{array}{l}\text { CBD inibiu GFAP, diminuiu } \\
\text { a expressão de proteínas } \\
\beta \text {-amilóide e regulou a ex- } \\
\text { pressão de iNOS e IL-1 } 1 \beta \text {. }\end{array}$ \\
\hline
\end{tabular}




\begin{tabular}{|c|c|c|c|c|c|}
\hline Estudo & Modelo & Tratamento & Variáveis analisadas & Método utilizado & Resultado \\
\hline $\begin{array}{l}\text { Sagredo et al. } \\
(2007)\end{array}$ & $\begin{array}{l}\text { Modelo de doença de Hunting- } \\
\text { ton: atrofia do estriado gerado } \\
\text { pela exposição tóxica de 3-NP }\end{array}$ & $\begin{array}{l}\text { Administração de } 10 \mathrm{mg} / \\
\mathrm{kg} \text { de CBD } 4 \text { horas depois } \\
\text { de feita cada injeção de } \\
\text { 3-NP }\end{array}$ & \begin{tabular}{|l|} 
Degeneração neuronal e \\
redução dos níveis de mRNA \\
de marcadores GABAérgicos
\end{tabular} & Hibridização in situ & $\begin{array}{l}\text { CBD diminuiu a atrofia neu- } \\
\text { ronal e reverteu às reduções } \\
\text { induzidas por } 3 N P \text { no conte- } \\
\text { údo de GABA }\end{array}$ \\
\hline $\begin{array}{l}\text { García- } \\
\text {-Arencibia et } \\
\text { al. (2007) }\end{array}$ & $\begin{array}{l}\text { Modelo de Doença de Parkin- } \\
\text { son: aplicação de 6-hidroxido- } \\
\text { pamina a nível cerebral }\end{array}$ & $\begin{array}{l}\text { Administração de CBD ( } 3 \\
\mathrm{mg} / \mathrm{kg} \text { ) logo após a aplica- } \\
\text { ção de } 6 \text {-hidroxidopamina } \\
\text { ou nas primeiras } 16 \mathrm{~h} \text { de- } \\
\text { correntes da aplicação. }\end{array}$ & $\begin{array}{l}\text { Níveis de mRNA para a su- } \\
\text { peróxido-dismutase e ROS }\end{array}$ & Hibridização in situ & $\begin{array}{l}\text { CBD regulou positivamente } \\
\text { os níveis de mRNA para a } \\
\text { superóxido-dismutase e foi } \\
\text { eficiente na diminuição de } \\
\text { ROS. }\end{array}$ \\
\hline $\begin{array}{l}\text { Castillo et al. } \\
(2010)\end{array}$ & $\begin{array}{l}\text { Modelo de hipóxia-isquêmica, } \\
\text { desenvolvida através da priva- } \\
\text { ção de oxigênio e glicose }\end{array}$ & $\begin{array}{l}\text { Administração de } 1 \text { a } 5 \mu \mathrm{M} \\
\text { de CBD na Cultura }\end{array}$ & $\begin{array}{l}\text { Níveis de caspase } 9 \text { e expres- } \\
\text { são de COX- } 2 \text { e iNOS }\end{array}$ & Espectrofluorimetria & $\begin{array}{l}\text { CBD diminuiu a morte celular, } \\
\text { inibiu caspase } 9, \text { COX-2 e iNOS } \\
\text { de maneira dose dependente. }\end{array}$ \\
\hline $\begin{array}{l}\text { Fagherazzi et } \\
\text { al. (2011) }\end{array}$ & $\begin{array}{l}\text { Modelo de doenças neuro- } \\
\text { degenerativa causado pela } \\
\text { deficiência cognitiva induzida } \\
\text { por sobrecarga de ferro }\end{array}$ & $\begin{array}{l}\text { Injeção intraperitoneal } \\
\text { aguda de CBD nas doses } \\
\text { de } 5,0 \text { ou } 10,0 \mathrm{mg} / \mathrm{kg} \text {, ou } \\
\text { injeções intraperitoneais } \\
\text { de CBD durantes } 14 \text { dias }\end{array}$ & $\begin{array}{l}\text { Uso crônico ou agudo de } \\
\text { CBD na recuperação do dano } \\
\text { cognitivo }\end{array}$ & $\begin{array}{l}\text { Treinamento de ratos } \\
\text { para a tarefa de reco- } \\
\text { nhecimento de novo } \\
\text { objeto. }\end{array}$ & $\begin{array}{l}\text { CBD: Uso crônico melhorou } \\
\text { a memória dos ratos em } \\
\text { relação ao teste de reconhe- } \\
\text { cimento do objeto. }\end{array}$ \\
\hline $\begin{array}{l}\text { Pazos et al. } \\
\text { (2012) }\end{array}$ & $\begin{array}{l}\text { Modelo de hipóxia-isquêmica, } \\
\text { desenvolvida através da priva- } \\
\text { ção de oxigênio e glicose }\end{array}$ & $\begin{array}{l}1 \mathrm{mg} / \mathrm{kg} \text { de CBD admi- } \\
\text { nistrado no sétimo e no } \\
\text { trigésimo dia após a lesão } \\
\text { neuronal }\end{array}$ & $\begin{array}{l}\text { Capacidade do CBD de recu- } \\
\text { perar a memória dos ratos } \\
\text { frente à realização de testes } \\
\text { comportamentais devido a } \\
\text { redução da lesão }\end{array}$ & $\begin{array}{l}\text { Técnicas de Western } \\
\text { Blotting e ressonância } \\
\text { magnética }\end{array}$ & $\begin{array}{l}\text { CBD reduziu o volume da } \\
\text { lesão em } 30 \% \text { sete dias após } \\
\text { a lesão e } 14 \% \text { no trigési- } \\
\text { mo quarto dia após a lesão } \\
\text { neuronal. }\end{array}$ \\
\hline $\begin{array}{l}\text { Silva et al. } \\
(2013)\end{array}$ & $\begin{array}{l}\text { Modelo de doença neurodege- } \\
\text { nerativa: dano cognitivo oca- } \\
\text { sionado pelo acúmulo de ferro } \\
\text { cerebral em ratos }\end{array}$ & $\begin{array}{l}30 \mathrm{mg} / \mathrm{kg} \text { de } \mathrm{Fe}^{2+} \text { do } 12^{\circ} \\
\text { ao } 14^{\circ} \text { dia pós-natal e } \\
\text { tratamento com CBD por } \\
14 \text { dias consecutivos na } \\
\text { fase adulta. }\end{array}$ & $\begin{array}{l}\text { A expressão gênica de } \\
\text { DNM1L no hipocampo e } \\
\text { OPA1 no córtex }\end{array}$ & Western blotting & $\begin{array}{l}\text { CBD reverteu os efeitos indu- } \\
\text { zidos pelo ferro, reduzindo } \\
\text { os níveis hipocampais de } \\
\text { DNM1L, Caspase } 3 \text { e sinap- } \\
\text { tofisina }\end{array}$ \\
\hline $\begin{array}{l}\text { Santos et al. } \\
(2015)\end{array}$ & $\begin{array}{l}\text { Modelo de doença de Parkin- } \\
\text { son: toxicidade celular induzida } \\
\text { pela exposição a níveis tóxicos } \\
\text { de MPP+. }\end{array}$ & $\begin{array}{l}1,5,10,25 \text { e } 50 \\
\mu \mathrm{M} \text { de } C B D\end{array}$ & $\begin{array}{l}\text { Neuritogênese, a ação dos } \\
\text { receptores de NGF (TrkA) e } \\
\text { das proteínas neuronais nos } \\
\text { mecanismos associados a } \\
\text { neuroproteção endógena. }\end{array}$ & Western blotting & $\begin{array}{l}\text { CBD protegeu contra a morte } \\
\text { celular e a perda de neurônios } \\
\text { induzida pela neurotoxina } \\
\mathrm{MPP}^{+} \text {em células } \mathrm{PC} 12\end{array}$ \\
\hline
\end{tabular}

Fonte: Elaborado pelo autor

O primeiro trabalho a demonstrar o potencial neuroprotetor e antioxidante do CBD foi realizado por Hampson et al. (1998). Nele, os autores utilizaram culturas de neurônios corticais de ratos expostos a níveis tóxicos do neurotransmissor excitatório glutamato, o que provocou a morte neuronal por estimulação de receptores como NMDAR (N-metil-D-Aspartato) e AMPA, os quais contribuem para a acentuação do estresse metabólico causado pelo acúmulo de cálcio intracelular. Foi verificado através de espectrofluorimetria que o CBD impede a morte celular, quando administrado entre 18 e 20 horas após a exposição da cultura ao glutamato. Além disso, o CBD diminuiu a estimulação dos receptores NMDAR e AMPA e mostrou-se mais eficiente do que a ação de antioxidantes clássicos, como e escorbato e o $\alpha$-tocoferol, pois foi capaz de reduzir a presença de espécies reativas de oxigênio (ROS). Tais resultados sugerem que o CBD possa mediar processos neuroprotetores e, principalmente antioxidantes em patologias como a Doença de Parkinson, no sentido de neutralizar ou minimizar a progressão da doença.

luvone et al. (2004) estudaram o efeito do CBD sobre a toxicidade induzida pelo peptídeo $\beta$-amilóide em células $P C 12$. Os autores quantificaram os danos celulares através da técnica de Western Blotting, evidenciando redução da sobrevivência celular devido ao aumento das espécies reativas de oxigênio (ROS), e peroxidação de lipídeos, bem como aumento da expressão de Caspase 3 (enzima chave na cascata de sinalização celular para apoptose), fragmentação de DNA e aumento do cálcio intracelular. O tratamento das células com CBD antes da exposição ao $\beta$-peptídeo aumentou significativamente a quantidade de células sobreviventes em relação ao grupo controle, que não recebeu o tratamento com CBD. Além disso, em todas as concentrações observou-se a diminuição da produção de ROS, da peroxidação lipídica, dos níveis de Caspase 3, da fragmentação do DNA, e diminuição do cálcio intracelular. Os resultados indicam que o CBD exerce, de forma combinada, efeitos neuroprotetores, anti-inflamatórios e anti-apoptótico, inibindo a Caspase 3 e atuando sobre a toxicidade do $\beta$-peptídeo, o que pode desempenhar um papel importante na neutralização ou diminuição da progressão da morte celular de neurônios, como ocorre, por exemplo, na doença de Alzheimer.

Esposito et al. (2006) investigaram as propriedades anti-inflamatórias do CBD em um modelo in vivo de neuroinflamação relacionada a Doença de Alzheimer em ratos. Os peptídeos $\beta$-amilóides humanos foram inoculados no hipocampo dos ratos e os mesmos foram tratados diariamente com CBD nas doses de 2,5 e $10 \mathrm{mg} / \mathrm{kg} \mathrm{du}-$ rante 7 dias. A presença de mRNA para a Proteína Glial Fibrilar Ácida (GFAP) foi avaliada através da hibridização in situ e a expressão de GFAP, iNOS e IL-1 $\beta$, que caracteristicamente são expressas em processos inflamatórios, foram determinadas pela análise de imunofluorescência. Em contraste com o grupo controle, nos animais tratados com CBD, observou-se que foi inibida a expressão de GFAP 
e seus mRNAs, assim como diminuíram a expressão de proteínas $\beta$-amilóide de maneira dose dependente. Além disso, o CBD ainda equilibrou os níveis de NO celular, devido à regulação da expressão da enzima iNOS e da proteína IL-1ß. O CBD mostrou-se eficiente na reversão e regulação de processos neuroinflamatórios, quando utilizado in vivo. Além disso, esses resultados mostram-se promissores como ferramenta farmacológica para atenuar a toxicidade celular e a neuroinflamação que induz morte neuronal nos processos neurodegenerativos.

Sagredo et al. (2007) estudaram o efeito neuroprotetor do CBD em um modelo de atrofia do estriado gerado pela exposição tóxica de 3-NP (Ácido 3- nitropropiônico). Os ratos utilizados foram submetidos a duas injeções por dia de 3-NP durante 5 dias e uma dose de $10 \mathrm{mg} / \mathrm{kg}$ de CBD 4 horas após cada injeção. Todos os animais foram mortos 90 min após receberem a última injeção e imediatamente seus cérebros foram removidos para avaliação neuroquímica do grau de lesão neuronal causada por 3-NP. Observou-se que a administração de 3-NP causou depleção significativa no conteúdo de GABA no estriado, acompanhada de redução paralela dos níveis de mRNA de vários marcadores GABAérgicos de neurônios estriados, resultando em processo de degeneração neuronal. Os animais que receberam as doses de CBD apresentaram acentuada diminuição da atrofia neuronal, pois o CBD reverteu completamente as reduções induzidas pela 3-NP no conteúdo GABA. Os resultados indicam que o CBD exerceu seus efeitos neuroprotetores agindo preferencialmente em neurônios do estriado que se projetam para a substância nigra, e que seu uso pode ter importante papel terapêutico nas patologias neurodegenerativas, como a doença de Huntington, caracterizada pela perda de neurônios estriados.

García-Arencibia et al. (2007) usaram um modelo experimental de Doença de Parkinson desenvolvido através da aplicação de 6-hidroxidopamina em nível cerebral, o que induziu a morte de grande parte dos neurônios nigroestriatais dopaminérgicos. O CBD foi capaz de recuperar a depleção induzida por 6-hidroxidopamina quando foi administrado imediatamente após a lesão, ou nas primeiras 16 horas após a lesão, porém não quando o tratamento foi iniciado uma semana depois da aplicação de 6-hidroxidopamina. Além disso, o efeito neuroprotetor e antioxidante do CBD deu-se pela regulação positiva dos níveis de mRNA para a superóxido-dismutase, uma enzima chave na defesa endógena contra o estresse oxidativo. Acredita-se que esse efeito foi independente de receptores e, ainda, que o CBD foi eficiente, na medida em que forneceu a neuroproteção contra a degeneração progressiva dos neurônios, sendo que sua principal contribuição foi induzir a diminuição dos níveis de radicais livres. Uma observação muito importante em relação a esse estudo é que o CBD pode ter papel preventivo no tratamento da Doença de Parkinson para pacientes que, por exemplo, sejam portadores de mutação no gene precursor da doença, visto que foi observado que ele é eficiente somente nos momentos iniciais da patologia.

Castillo et al. (2010) também relataram as propriedades neuroprotetoras do CBD, investigando sua ação em um modelo in vitro de hipóxia-isquêmica, desenvolvido em fatias do córtex frontal de cérebros de ratos recém-nascidos através da privação de oxigênio e glicose (OGD). Os resultados indicam que o CBD diminuiu a morte celular através da inibição da Caspase 9, e ainda que ele foi capaz de modular a liberação de glutamato e a expressão de COX-2 e iNOS, duas importantes enzimas presentes em processos de inflamação e citotoxicidade. Os autores observaram ainda que os principais receptores que mediaram a ação do CBD foram o receptor canabinóide do tipo 2 (CB2) e receptores de adenosina, principalmente do tipo A2A. Os resultados contribuem para o melhor entendimento de como o CBD melhora a função cerebral, e consequentemente é capaz de recuperar lesões no sistema nervoso central, podendo ser empregado para melhorar a função cognitiva após processos lesivos.

Fagherazzi et al. (2011) estudaram os efeitos do CBD utilizando um modelo animal de deficiência cognitiva induzida por sobrecarga de ferro, a fim de testar seus efeitos no tratamento de déficits cognitivos associados à presença de patologias neurodegenerativas. Para tanto, ratos foram preliminarmente treinados para a tarefa de reconhecimento de novo objeto. Após o período de treinamento, receberam injeções intraperitoneais de ferro nos dias pós-natais 12-14. Com a idade de 2 meses, parte dos ratos recebeu uma injeção intraperitoneal aguda de CBD nas doses de 5,0 ou 10,0 mg / kg, e foram novamente submetidos a sessões de treino para tarefa de reconhecimento do novo objeto. O restante dos ratos, a fim de investigar os efeitos do uso crônico do CBD, receberam injeções intraperitoneais de CBD durantes os próximos 14 dias e, ao final do tratamento, foram também submetidos novamente ao teste de reconhecimento de novo objeto. Foi observado que uma única injeção de CBD na dose mais elevada foi capaz de recuperar parcialmente a memória de ratos tratados com ferro. Evidenciou-se ainda que, com o tratamento crônico, a memória dos ratos melhorou em relação ao teste de reconhecimento do objeto, indicando que foi possível reverter os danos cognitivos induzidos pelo acúmulo de ferro quando o tratamento com CBD foi aplicado de forma contínua. Além disso, doses de CBD agudas e crônicas não tiveram influência na memória dos ratos do grupo controle. Os resultados sugerem um possível uso do CBD para tratamento ou prevenção de quadros de déficits cognitivos associados à presença de doenças neurodegenerativas.

Pazos et al. (2012) avaliaram o efeito neuroprotetor do CBD em um modelo experimental de hipóxia cerebral isquêmica, utilizando ratos Wistar neonatos, através da privação de oxigênio e glicose e redução do fluxo sanguíneo. A hipóxia isquêmica leva a um prejuízo funcional e cognitivo. Nos primeiros 7 e 30 dias após a lesão, todos os ratos foram submetidos a testes neurocomportamentais tais como rotarod, teste de cilindro traseiro (CRT) e reco- 
nhecimento de objetos (NOR). Dessa forma, observou-se que os ratos tratados com CBD apresentaram melhor comportamento funcional em relação à execução das atividades, tanto no sétimo dia quanto após o primeiro mês decorrente do tratamento. Tal fato indica que o CBD exerceu sua ação neuroprotetora de forma prolongada e efetiva. Os autores ainda demostraram, utilizando as técnicas de Western Blotting e ressonância magnética, que o CBD reduziu o volume da lesão em $30 \%$ sete dias após a lesão e $14 \%$ no trigésimo quarto dia após a lesão neuronal. A análise histológica mostrou que o CBD está associado à modulação do estresse oxidativo, inflamação e excitotoxidade. Além disso, seu uso não foi associado a efeitos colaterais. Foi sugerido neste trabalho que o CBD pode atuar como agente preventivo, pois nos ratos tratados no sétimo dia após a lesão o percentual de recuperação foi maior do que quando o tratamento foi administrado mais tarde.

Silva et al. (2013) analisaram o efeito do CBD na reversão dos danos causados pelo acúmulo de ferro nos cérebros de ratos, que receberam $30 \mathrm{mg} / \mathrm{kg}^{\mathrm{de} \mathrm{Fe}} \mathrm{Fe}^{2+}$ do $12^{\circ}$ ao $14^{\circ}$ dia pós-natal. Os níveis proteicos e a expressão gênica no hipocampo e córtex foram quantificados através de Western blotting, e constatou-se que, logo após o tratamento com ferro, houve redução de DNM1L no hipocampo e OPA1 no córtex, proteínas importantes na regulação e controle dos processos de fissão e fusão mitocondrial. Além disso, o ferro induziu um aumento nos níveis de Caspase 3 tanto no hipocampo quanto no córtex, acompanhado por redução dos níveis de sinaptofisina, o que levaria a perda sináptica e a morte celular. Quando os ratos alcançaram a idade adulta, foram tratados com CBD durante 14 dias e mortos 24 horas após a última injeção para análise do hipocampo e córtex cerebral. Foi observado através de Western blotting que o CBD reverteu os efeitos induzidos pelo ferro, trazendo os níveis hipocampais de DNM1L, Caspase 3 e sinaptofisina de volta aos valores comparáveis ao grupo controle. Os resultados sugerem que o ferro afetou a dinâmica mitocondrial e desencadeou perda sináptica e morte celular por apoptose, o que foi revertido pelo tratamento com CBD, indicando que este exerceu atividade neuroprotetora e pode ser um importante agente terapêutico em casos de degeneração neuronal causada pelo acumulo de ferro, como é característico na Doença de Parkinson e Alzheimer.

Santos et al. (2015) estudaram os efeitos neuroprotetores do CBD em um modelo de toxicidade celular, em que células PC12 de ratos foram expostas a níveis tóxicos de $\mathrm{MPP}^{+}$. Foram investigados os fatores relacionados à neuritogênese, a ação dos receptores de NGF (TrkA) e das proteínas neuronais nos mecanismos associados a neuroproteção endógena desencadeada contra a toxicidade $\mathrm{MPP}^{+}$nas células. $\mathrm{O}$ teste Western blotting evidenciou que o CBD protege contra a morte celular e a perda de neurônios induzida pela neurotoxina $\mathrm{MPP}^{+}$em células
PC12. A neuroproteção não é dependente ou mediada pelo NGF, sendo que pode estar associada a receptores TrkA e o aumento da expressão de proteínas neuronais. Entretanto, o CBD parece não ativar receptores TrkB e TrkC. Tais resultados mostram-se importantes principalmente no que diz respeito à doença de Parkinson, pois nesta patologia ocorre com frequência influência do $\mathrm{MMP}^{+}$na citotoxicidade neuronal.

Todos os estudos incluídos nesta revisão mostraram que o CBD exerceu efeitos benéficos quando administrado em modelos experimentais de doenças neurodegenerativas. Esses efeitos foram primeiramente pautados na eficácia do CBD em reduzir a presença de ROS e iNOS, mediando processos antioxidante e neuroprotetores e também em sua capacidade de inibição das enzimas associadas à apoptose, como a caspase 3 a e caspase 9 , minimizando a perda neuronal e um possível declínio cognitivo associado a perda sináptica. Também foi possível observar que os mecanismos de ação do CBD deram-se devido a sua interação com diversos neurotransmissores e neuromoduladores, como por exemplo, o conteúdo de GABA, assim como foi capaz de mediar à inibição de importantes proteínas presentes em processos neuroinflamatórios e degenerativos.

A molécula ainda foi capaz de melhorar a função cerebral e recuperar significativamente lesões existentes no sistema nervoso, sendo apontada em vários dos estudos incluídos nesta revisão como uma molécula com um possível papel na prevenção de quadros de déficits cognitivos induzidos ou provocados pelo desenvolvimento de patologias neurodegenerativas.

\section{CONCLUSÕES}

A revisão mostrou que o CBD é uma molécula promissora para o tratamento de doenças neurodegenerativas. No entanto, seus mecanismos de ação são diversos e não estão totalmente elucidados. 0 que se sabe até o momento é que a molécula exerce suas propriedades neuroprotetoras através do equilíbrio de fatores relacionados à toxicidade celular que pode levar à morte neuronal gradual que com o passar do tempo evolui para uma patologia neurodegenerativa.

Mesmo considerando os aspectos promissores da molécula, há a necessidade de reforçar esses estudos para melhor compreender seus mecanismos de ação e os seus possíveis efeitos colaterais. Estudos clínicos relacionados à ação e interação do CBD em doenças neurodegenerativas não foram encontrados o que sugere cautela ao transpor os resultados para humanos.

\section{REFERÊNCIAS}

CASTILLO, A. et al. The neuroprotective effect of cannabidiol in an in vitro model of newborn hypoxic-ischemic brain damage in mice is mediated by CB2 and adenosine receptors. Neurobiol. dis., Orlando, v. 37, n. 2, p.434-440, 2010

CRIPPA, J. A. et al. Cannabis and anxiety: a critical review of the evidence. Hum. psychopharmacol., Chichester, v. 24, n. 7, p. 515-523, 2009. 
ESPOSITO, G. et al. Cannabidiol inhibits inducible nitric oxide synthase protein expression and nitric oxide production in $\beta$-amyloid stimulated PC12 neurons through p38 MAP kinase and NF-?B involvement. Neurosci. lett., Amsterdam, v. 399, n. 1, p. 91-95, 2006.

FAGHERAZZI, E. V. et al. Memory-rescuing effects of cannabidiol in an animal model of cognitive impairment relevant to neurodegenerative disorders. Psychopharmacol., Berlin, v. 219, n. 4, p.1133-1140, 2011.

GARCÍA-ARENCIBIA, M. et al. Evaluation of the neuroprotective effect of cannabinoids in a rat model of Parkinson's disease: Importance of antioxidant and cannabinoid receptor-independent properties. Brain res., Amsterdam, v. 1134, p.162-170, 2007.

HAMPSON, A. J. et al. Cannabidiol and (-) 9-tetrahydrocannabinol are neuroprotective antioxidants. Proc. Natl. Acad. Sci. U.S.A., Washington, v. 95, n. 14, p.8268-8273, 1998.

IUVONE, T. et al. Neuroprotective effect of cannabidiol, a non-psychoactive component from Cannabis sativa, on beta-amyloid-induced toxicity in PC12 cells. J. neurochem., Oxford, v. 89, n. 1, p.134-141, 2004.

JOSEPH, J. et al. Nutrition, brain aging, and neurodegeneration. J. Neurosci., Baltimore, v. 29, n. 41, p.12795-12801, 2009.

MAGALHÃES, J. P; SANDBERG, A. Cognitive aging as an extension of brain development: A model linking learning, brain plasticity, and neurodegeneration. Mech. ageing dev., Limerick, v. 126, n. 10, p.1026-1033, 2005.

MECHOULAM, R. et al. Chemical Basis of Hashish Activity. Science, Washington, v. 169, n. 3945, p. 611-612, 1970.

NUSSBAUM, R. L; MCINNES, R. R; WILLARD, H. F. Genética Médica. 7 ed. Rio de Janeiro: Elsevier, 2008.
PAZOS, M. et al. Cannabidiol administration after hypoxia-ischemia to newborn rats reduces long-term brain injury and restores neurobehavioral function. Neuropharmacology, Oxford, v. 63, n. 5, p.776-783, 2012.

PEREZ, M. Papel neuroprotetor do canabidiol em ratos neonatos após transecção do nervo isquiático. 2013. Dissertação (Mestrado) - Curso de Programa de Pós-graduação em Biologia Celular e Estrutural, Instituto de Biologia, Universidade Estadual de Campinas, Campinas, 2013.

SAGREDO, O. et al. Cannabidiol reduced the striatal atrophy caused 3-nitropropionic acid in vivo by mechanisms independent of the activation of cannabinoid, vanilloid TRPV1 and adenosine A2A receptors. Eur. j. neurosci., Oxford, v. 26, n. 4, p. 843-851, 2007.

SANTOS, N. A. G. et al. The neuroprotection of cannabidiol against MPP+ induced toxicity in PC12 cells involves TrkA receptors, upregulation of axonal and synaptic proteins, neuritogenesis, and might be relevant to Parkinson's disease. Toxicol. in vitro, New York, v. 30, n. 1, p. 231-240, 2015.

SCUDERI, C. et al. Cannabidiol in medicine: a review of its therapeutic potential in CNS disorders. Phytother. res., London, v. 23, n. 5, p.597602, 2009.

SILVA, V. K. et al. Cannabidiol Normalizes Caspase 3, Synaptophysin, and Mitochondrial Fission Protein DNM1L Expression Levels in Rats with Brain Iron Overload: Implications for Neuroprotection. Mol. neurobiol., New York, v. 49, n. 1, p. 222-233, 2013.

THE GLOBAL impact of dementia: an analysis of prevalence, incidence, cost and trends. London: Alzheimer's Disease International, 2015.

ZUARDI, A. W. Cannabidiol: from an inactive cannabinoid to a drug with wide spectrum of action. Rev. Bras. Psiquiatr., São Paulo, v. 30, n. 3, p.271-280, 2008.

Submetido em: 22/12/2016

Aceito em: 21/05/2017 